\title{
Una aproximación psicosocial al conflicto vasco como sistema dinámico*
}

\section{A Psychosocial Approach to the Basque Conflict as a Dynamical System}

Recibido: 30 de abril de 2013 | Revisado: 5 de febrero de 2015 | Aceptado: 5 de febrero de 2015

\author{
XAVIER MíNGUEZ ALCAIDE ** \\ Ramón Alzate Sáez de Heredia *** \\ MANUEl SÁNCHEZ DE MIGUEL ***** \\ Universidad del País Vasco/Euskal Herriko \\ Unibertsitatea, España
}

doi:10.11144/Javeriana.upsy14-2.apcv

Para citar este artículo: Mínguez Alcaide, X., Alzate, R., \& Sánchez de Miguel, M. (2015). Una aproximación psicosocial al conflicto vasco como sistema dinámico. Universitas Psychologica, 14(2), 563-578. http://dx.doi.org.10.11144/Javeriana.upsy14-2.apcv

* Artículo de investigación: realización entre abril de 2009 y junio de 2010.

Agradecimientos: A la Universidad del País Vasco por la concesión de la ayuda predoctoral a Xavier Mínguez Alcaide y del Proyecto de Investigación "Métodos de Diálogo con Grandes Grupos para la Construcción de Paz en Euskadi".

** Investigador. Doctor. Colaborador en la cátedra de Resolución de Conflictos de la Facultad de Psicología de la Universidad del País Vasco. Contacto principal para correspondencia editorial: Avenida de Tolosa, 70. Donostia-San Sebastián (Gipuzkoa) 20018, España. Correo electrónico: xavier.minguez@ehu.es

*** Catedrático de Resolución de Conflictos. Correo electrónico: ramon.alzate@ehu.es

**** Correo electrónico: manu.sanchez@ehu.es

\section{RESUMEN}

El artículo presenta una aproximación psicosocial al conflicto del País Vasco desde el modelo dinámico/sistémico del conflicto (Coleman, 2006). La investigación se llevó a cabo a partir de 60 entrevistas en profundidad realizadas a personas de distintos sectores de la sociedad vasca, analizadas a partir de un análisis de contenido por categorización, y de un análisis interpretativo fenomenológico. Los resultados apuntan a cinco elementos centrales del conflicto: las manifestaciones de violencia y sus formas de legitimación, la vulneración de necesidades humanas básicas, la interdependencia negativa de las identidades colectivas, cuestiones políticas e ideológicas. Igualmente, se mencionan tres factores adicionales - polarización social, emociones y aspectos cognitivos-. Finalmente, se interpreta el conjunto de datos sobre el conflicto vasco a partir del modelo dinámico/sistémico.

Palabras clave

conflicto vasco; modelo dinámico sistémico del conflicto intratable; investigación cualitativa; análisis interpretativo fenomenológico

\section{A B S T R A C T}

The paper presents a psychosocial approach to Basque Country conflict from the dynamical system model of conflict (Coleman, 2006). Research was make throughout 60 interviews fulfilled with people of different sectors of basque society, and were analyzed by categorization content analysis and by interpretative fhenomenologycal analysis. Results point to five central elements of conflict: violence manifestations and there legitimation forms, violation of Basic Human Needs, negative interdependence of collective identities, political and ideological issues. Three additional factors are also mentionated -social polarization, emotional and cognitive issues-. Finally, we explain the whole dates about basque conflict throughout dynamic/ systemic model.

Keywords

basque conflict; dynamical system model of intractable conflict; qualitative research; interpretative phenomenologycal analysis 


\section{Introduccion}

\section{El modelo del conflicto intratable como un sistema dinámico}

El modelo psicosocial del conflicto como sistema dinámico introduce el marco interpretativo de los sistemas complejos para abordar los conflictos profundos (Coleman 2003, 2004a, 2006; Coleman, Vallacher, Nowak, \& Bue Ngoc, 2007; Musallam, Coleman, \& Nowak, 2010; Vallacher, Coleman, Nowak, \& Biu-Wrzosinska, 2010). Según esta perspectiva, los conflictos son estructuras multidimensionales dinámicas, es decir, redes complejas compuestas por muchos elementos interconectados que cambian y evolucionan con el tiempo pero sin perder su estructura sistémica. En tanto que sistemas complejos, los conflictos se perpetúan como consecuencia de las dinámicas relacionales que mantienen sus elementos, unas dinámicas marcadas por la multicausalidad y la autoorganización (Coleman et al., 2007; Dimitrov, 2000, 2003; Goerner, 1995; Johnson, 2003; Kauffman, 1993, 1995; Morales La Paz, 2003; Morin, 1995; Munné, 2005; Pérez Martínez, Nuño de la Rosa, \& González Recio, 2006).

El elemento clave del modelo desarrollado por Coleman y colaboradores es la noción de atractor (Coleman, 2006, 2012; Coleman et al., 2007; Vallacher et al., 2010; Novak, Bui-Wrzosinska, Vallacher, \& Coleman, 2012). De manera sintética, podemos decir que los atractores del conflicto 'son elementos psicológicos, sociales y estructurales muy estables y robustos, esto es, muy resistentes a ser modificados, y que están fuertemente vinculados entre sí y con otros elementos definitorios del conflicto, sobre los que tienen gran influencia'. Desde la óptica de los sistemas dinámicos, los atractores son los elementos que hacen de los conflictos profundos sistemas en equilibrio, y por lo tanto, muy difíciles de transformar. A nivel psicosocial, la fuerza de los atractores se refleja en la estabilidad de las orientaciones cognitivas, afectivas y conductuales que mantienen los diferentes actores del conflicto, y que son la base de la permanencia del conflicto.
Como hemos visto, los atractores son elementos de tipo psicológico, social y estructural. Los de tipo psicológico, pueden hacer referencia a patrones cognitivos distorsionados y polarizados y a índices bajos de complejidad cognitiva, a climas emocionales negativos o memorias colectivas construidas socialmente para la salvaguarda de la propia identidad de grupo. Por su parte, los de tipo social pueden ser las relaciones intergrupales hostiles, los patrones de socialización y de construcción colectiva de significados sobre el conflicto o los mecanismos asociados a la polarización social. Finalmente, los atractores de tipo estructural pueden hacer referencia a relaciones de poder asimétricas o a una historia de dominación política y cultural de un grupo sobre otro(s).

Esta perspectiva del conflicto basada en la complejidad es complementada por Coleman (2000, 2003, 2004b) con su propuesta de modelo integrado del conflicto etnonacional, donde incluye gran cantidad de factores psicológicos, sociales y estructurales que, relacionados entre sí de manera multilineal, funcionan como un sistema complejo.

Un modelo psicosocial integrado de los conflictos etnonacionales

Los conflictos etnonacionales son aquellos donde un grupo nacional y/o minoritario reclama una mayor soberanía política al Estado nacional (Gurr, 2000) y donde las identidades colectivas adquieren un papel preponderante (Bar-Tal, 2007; Bar-Tal \& Rouhana, 1998; Brown, 1997; Coleman, 2000, 2003, 2004b; Horowitz, 1985; Kelman, 1997, 1999; Rothman, 1997; Rouhana, 2004; Smith, 1993). La amenaza de las identidades colectivas por parte de los grupos enfrentados favorece la interdependencia negativa de dichas identidades, es decir, la percepción según la cual la supervivencia de la propia identidad colectiva solo es posible a costa de la identidad del Otro (Kelman, 1997; 1999).

El modelo psicosocial integrado del conflicto etnonacional propuesto por Coleman (2000; 2003; 2004b), contempla elementos psicológicos, sociales y estructurales. Entre las condiciones 
estructurales destacan: la existencia de unas relaciones históricas de enemistad y/o de dominación estructural y cultural, unas condiciones de vida difíciles (Druckman, 2001; Gurr, 2000; Isajiw, 2000; Lederach, 1998), la vulneración de Necesidades Humanas Básicas (NHB) (Azar, 1983; 1990; Burton, 1987; 1990a; Doob, 1970; Fisher, 1990; 1997; Kelman, 1986; 1992; 1997) o una contradicción profunda asociada a cuestiones existenciales y valores culturales no negociables (Burton, 1990b).

Entre los procesos sociales, podemos destacar: a) la polarización social, con el desarrollo de estructuras sociales exclusivas, excluyentes y aislacionistas (Coleman, 2003; Martín-Baró, 1983), b) el bloqueo de la comunicación social y el silencio social (Martín Beristain \& Páez, 2000; Overschall, 2000), c) las formas de liderazgo militante y estrategias de línea dura, d) los patrones de socialización donde los individuos desarrollan sus identidades y visiones acerca de los diferentes dominios del conflicto (Toscano, 1998) y e) el etnocentrismo y las ideologías nacionalistas (Korostelina, 2004, 2007).

A nivel psicosocial, indica la existencia de elementos cognitivos, perceptivos, afectivos, morales y conductuales que conforman la infraestructura psicosocial subyacente al conflicto etnonacional. A nivel cognitivo y perceptivo, destaca la relevancia de bajos índices de complejidad cognitiva, las imágenes especulares (Bronfenbrenner, 1961; Kelman, 1997), o memorias colectivas enfrentadas que son centrales para la construcción de las identidades colectivas (Aravena, 2003; Bar-Tal, 2007; Halbwchs, 2004; Páez, Valencia, Pennebaker, Rimé, \& Jodelet, 1998; Rosa Rivero, Bellelli, \& Bakhurst, 2000). A nivel actitudinal y conductual, señala la discriminación y hostilidad hacia el exogrupo, las posiciones extremas y de línea dura y los mecanismos de cohesión y apego a la normatividad grupal (Coleman, 2004b; Rubin, Pruitt, \& Kim, 1994). A nivel afectivo, apunta a una gran intensidad y un apego emocional con el conflicto y al desarrollo de orientaciones emocionales colectivas negativas hacia el exogrupo y positivas hacia el propio (Bar-Tal, 2007; Blank \& Schmidt,
2003; Coleman, 2004b; Federico, Golec, \& Dial, 2005; Feshback, 1994; Worchel \& Coutant, 1997). En relación a las cuestiones morales, señala la importancia de los procesos de exclusión moral (Martín Peña \& Opotow, 2011; Opotow, 1990, 2005), deshumanización (Haslam, 2006) y desenganche moral (Bandura, 1999; Bandura, Barbaranelli, Caprara, \& Pastoreli, 1996).

\section{El caso de estudio: el conflicto del País Vasco}

El conflicto del País Vasco puede considerarse un fenómeno etnonacional de gran complejidad, definido por su historia, por cuestiones políticas e ideológicas, por identidades colectivas enfrentadas y por elevadas dosis de violencia en diferentes etapas (Gurr, 2000). A nivel histórico y político, el conflicto vasco presenta un desencuentro prolongado acerca de la soberanía en los territorios vascos (Apalategi, 1992, 2006). La manifestación contemporánea del conflicto vasco está vinculada a la actividad armada de Euskadi Ta Askatasuna (ETA), componente militar del Movimiento de Liberación Nacional Vasco (MLNV). ETA nace en 1959 como continuación de la organización estudiantil de carácter nacionalista EKIN (Alvarez Enparantza, 1997), y desarrolla su ideario influenciada por la ideología de los movimientos de liberación nacional de los años 50-60 (Bilbao, 1996). Tras 50 años de existencia, y con más de 850 muertes perpetradas, el 10 de enero de 2011 ETA envía un video y un comunicado al diario Gara en el que declara un alto el fuego permanente y de carácter general, que puede ser verificado por la comunidad internacional (ETA, 2011).

Partiendo de la concepción del conflicto como sistema dinámico, donde unos atractores dan estabilidad al conflicto como sistema, esta investigación se propuso examinar cuales son los atractores centrales del conflicto vasco desde una perspectiva psicosocial. Así, la pregunta que nos sirvió como punta de partida para el desarrollo de esta investigación fue: icuáles son los elementos centrales del conflicto vasco que permiten su perpetuación? 


\section{Método}

\section{Diseño}

Para aproximarnos a los elementos centrales del conflicto vasco, desde una óptica psicosocial, se estableció un diseño de investigación inductivoexploratorio de carácter cualitativo, basado en el uso de dos herramientas de análisis: el Análisis de Contenido por Categorización (Bardin, 1996; Hostil, 1969) y el Análisis Interpretativo Fenomenológico (IPA) (Smith, 2003, 2008; Smith, Harré, $\&$ Van Langehnove, 1995). Con el objeto de obtener múltiples significados (Bruner, 1991) que nos permitieran obtener una visión global del conflicto y acercarnos a sus atractores, se desarrolló un modelo de entrevista semiestructurada centrada en varias áreas de interés, como son: a) la saliencia del conflicto a nivel individual, social y político, b) los aspectos considerados centrales del conflicto, aquellos que han dado origen al mismo y los que han facilitado su perpetuación, c) el contacto intergrupal, la comunicación social y la polarización social y d) los sentimientos y las vivencias emocionales en relación con el conflicto. Para la obtención de significados acerca de estas áreas de interés, se planteó un guión de entrevista fenomenológica (Smith, 2003, 2008; Smith et al., 1995) a partir de una serie de preguntas.

\section{Participantes}

En la investigación participaron 60 personas (27 mujeres y 33 hombres) de diferentes sectores de la sociedad vasca designados como ámbitos sociales de interés. Estos ámbitos sociales fueron: a) orga- nizaciones y movimientos sociales (sindicalismo, paz y DD. HH., cooperación al desarrollo, víctimas de ETA, presos de ETA y construcción nacional, otros); b) educación; c) periodismo; d) sanidad y e) funcionariado. Los/as participantes fueron clasificados según su adscripción ideológica y nacionalista, obteniéndose un $3 \%$ de nacionalistas españoles de ideología conservadora (NEC), un 32 \% de nacionalistas españoles de ideología progresista (NEP), un $23 \%$ no nacionalista (NN), un $30 \%$ de nacionalistas vascos moderados ${ }^{1}$ (NVM) y un $10 \%$ de nacionalistas vascos radicales ${ }^{2}$ (NVR). El rango de edad de los participantes fue de entre 25 y 71 años, con una media de edad de 42.

\section{Procedimiento}

Con anterioridad al desarrollo de las entrevistas, se llevó a cabo una preparación intensiva del entrevistador. Las 60 entrevistas fueron conducidas por el mismo entrevistador y se implementaron cara a cara, bien en la Facultad de Psicología de la Universidad del País Vasco, o en los puestos de trabajo o centros de las organizaciones de los/as participantes. El intervalo de duración de las entrevistas fue de entre 27 y 112 minutos, con una duración media de 47 minutos. Todas las entrevistas fueron grabadas en audio y transcritas en su totalidad.

Las entrevistas se desarrollaron siguiendo los patrones del IPA (Smith, 2003, 2008; Smith et al., 1995). Así, se puso el foco en los temas de interés de los investigadores, pero con la suficiente flexibilidad para dar entrada a cuestiones emergentes

1 Nacionalistas vascos no vinculados al MLNV.

2 Nacionalistas vascos vinculados al MLNV.

\section{Cuadro 1}

Guion de las entrevistas

- ¿Qué importancia tiene para ti la situación de violencia y conflicto que se vive en el País Vasco?

- ¿Cuáles son los aspectos centrales del conflicto, aquellos que dieron origen al mismo y los que hacen que se perpetúe?

- ¿Mantienes contacto con personas que tienen puntos de vista diferentes al tuyo?, ¿Habláis acerca del conflicto y la paz en el País Vasco?, ¿Qué sucede a nivel social respecto a estas cuestiones?

- ¿Qué sentimientos te provoca la situación de conflicto?, ¿Qué emociones podrías vincular a diferentes situaciones del conflicto?, ¿Cuáles crees que son las emociones que determinan el clima emocional de la sociedad vasca?

Fuente: Elaboración propia 
planteadas por los/as participantes, facilitando la aportación de significados sobre el conflicto y la paz en el País Vasco. Por otro lado, las entrevistas se implementaron a partir de las preguntas generales (Cuadro 1), dando paso a preguntas más concretas en función de las narrativas, acompañando a cada participante en la construcción de su discurso, mediante las técnicas de reflejo, reformulación y reinterpretación del discurso (Community Boards \& Alzate, 2000), tanto en relación con los ámbitos de interés como sobre las cuestiones emergentes planteadas por ellos/as mismos.

\section{Análisis de los datos}

Con el fin de clasificar los datos obtenidos, se estableció un sistema de categorías, partiendo de las variables indicadas por Coleman (2000, 2003, 2004b) y apoyándonos en la literatura de referencia sobre el conflicto etnonacional (Azar, 1990; Bar-Tal, 2007; Burton, 1987, 1990a, 1990b; Christie, Wagner, \& Winter, 2001; Deutsch, 1973; Fisher, 1990, 1997; Galtung, 1969, 1990, 2003; Gurr, 2000; Horowitz, 1985; Kelman, 1997, 1999; Lederach, 1995, 1998; Ross, 1995, 1997; Rothman, 1997; Rouhana, 2004; Rubin et al., 1994). Como resultado esta revisión, se compuso un sistema de 190 categorías, divididas en 13 grandes bloques: 1) cuestiones estructurales, vulneración de las NHBy violencia; 2) polarización social; 3) contacto y comunicación intergrupal; 4) interdependencia negativa de las identidades colectivas; 5) procesos de liderazgo; 6) procesos de socialización; 7) procesos cognitivos y perceptivos; 8) actitudes y comportamientos; 9) sistemas de creencias; 10) dilemas del conflicto; 11) memorias colectivas sobre el conflicto; 12) procesos afectivos y 13) procesos morales.

Cada una de las categorías fue definida operacionalmente para clasificar los datos obtenidos mediante un análisis de contenido por categorización (Bardin, 1996; Hostil, 1969). Este se realizó con la herramienta informática WINMAX y a partir de un acuerdo interjueces con el apoyo de un juez experto, obteniéndose un índice Kappa $=0.89 ; p$ $<0.001$. Una vez ordenados los datos en función de las categorías incluidas en los grandes bloques, se llevó a cabo la segunda fase de análisis mediante la técnica del IPA (Smith, 2003, 2008; Smith et al., 1995), con la finalidad de dar sentido al conjunto de significados obtenidos y aportar una interpretación focalizada en los atractores centrales del conflicto y la paz en el País Vasco.

\section{Resultados}

Los resultados del estudio se presentan siguiendo la interpretación de los atractores centrales del conflicto. Debido al elevado número de cuestiones vinculadas a los atractores del conflicto, en el presente artículo se presentarán los resultados sobre los cinco atractores centrales del conflicto vasco y algunos elementos asociados, y tres cuestiones adicionales consideradas de importancia. Estas tres cuestiones adicionales hacen referencia a la polarización social, a formas cognitivas y perceptivas rígidas y simplificadas, y al clima emocional.

\section{Polarización social borrosa}

Los resultados apuntan a una polarización social relativa en la sociedad vasca. Se señala que la mayoría social se encuentra en un intervalo de posiciones intermedias o moderadas, con grupos altamente polarizados vinculados al independentismo vasco que ha apoyado a ETA y al centralismo español de ideología conservadora. Vinculada a esta polarización social relativa, se enfatiza la existencia de una presión social de posicionarse en uno de los polos, y la visión negativa de las posiciones intermedias (Martín Baró, 1983). En este sentido, P18 (mujer, periodismo, NEP) afirma que:

Las posiciones más radicales son las que marcan ese juego. Parece que solo hay dos actores, pero hay muchos más, y la mayoría, mucha gente no se identifica ni con los unos ni con los otros. Ni con los que abogan por la lucha armada, o los que dicen que esto forma parte de España, no hay un pueblo, no hay una cultura. Yo creo que ni unos ni otros, que la mayoría de la gente estamos entre medio.

Aunque los resultados apuntan a la existencia de un índice elevado de contacto intergrupal, debido 
principalmente a la estructura social heterogénea en los territorios vascos y a una no segregación en comunidades enfrentadas, los resultados también indican cómo el entorno social del MLNV se ha desarrollado como un espacio social exclusivo, excluyente y aislacionista (Coleman, 2003). P51 (varón, educación, NVM) dice que:

Es muy endogámico, muy difícil de entrar, de no ser sospechoso de entrar. También no necesitas salir, porque aunque sea un 15 o un $12 \%$, o igual en un pueblo es un $30 \%$, tienen mucha gente, o sea, puedes vivir en su mundo sin relacionarte con nadie más.

Por otro lado, y a pesar de la existencia de un elevado contacto intergrupal, de los resultados se desprende la existencia de una baja comunicación social sobre el conflicto (Coleman, 2004b; Overschall, 2000), un silencio social (Martín-Beristain \& Páez, 2000) que es debido al tabú producido por el miedo social, a conductas evitativas en el medio social y al hecho de no hablar sobre el conflicto para no dañar relaciones familiares y de amistad.

\section{Rigidez y simplificación cognitiva, imagen especular y narrativas del conflicto}

Los patrones de pensamiento y percepción en situaciones de conflicto suelen estar marcados por la rigidez y la simplificación (Coleman \& Lowe, 2007; Conway, Suedfeld, \& Tetlock, 2001; Martín Baró, 1983). En este sentido, los resultados indican la existencia de una baja complejidad cognitiva, con una baja integración de las diferentes cuestiones y de aplicación de diferentes marcos de interpretación del conflicto vasco, como determinantes del conflicto. Además, se señala la existencia de una baja tolerancia a la información contradictoria y una introyección de la información basada en la identificación y la conformidad con las estructuras de liderazgo. P1 (mujer, movimientos sociales otros, NEP) afirma:

Es una falta de capacidad de integración de diferentes visiones. Y eso necesita una ampliación de visión capaz de contemplar diferentes perspectivas, y ver en qué parte cada una tiene una parte integrable, e integrarla. Y eso es un problema de visión.

En este punto adquiere especial relevancia el tratamiento del conflicto vasco por parte de los medios de comunicación. Los resultados apuntan que estos son un actor importante del conflicto, ya que están vinculados a grupos políticos e ideológicos, manifestando sus puntos de vista y defendiendo sus intereses y, por tanto, siendo una influencia principal en la construcción de significados sobre el conflicto, tanto en el País Vasco como en el resto de España. P18 (mujer, periodismo, NEP) dice:

Sí es verdad, los medios de comunicación, si diéramos una información mucho mejor tratada y mucho más coherente, la gente no pensaría como piensa. La información sobre el conflicto vasco está muy manipulada. Muy, muy manipulada.

Asimismo, en los discursos se señala la presencia de imágenes de los grupos estereotípicas y esencialistas y de la existencia de imágenes especulares entre los grupos enfrentados (Bronfenbrenner, 1961; Kelman, 1997). P19 (mujer, educación, NEP) apunta que:

Si la otra persona percibe que soy una facha, y posiblemente yo que es una persona borroka $a^{3}$, fanática, en fin, yo creo que nos acabamos viendo igual. La percepción que va a tener de mí es de una persona facha en el sentido de fanática, y yo también en el otro extremo, acabo viendo una persona fanática, intransigente.

Por otro lado, un aspecto psicosocial central de los conflictos etnonacioales es la construcción de memorias colectivas vinculadas a las identidades colectivas y a intereses políticos (Bar-Tal, 2007; Deutsch, 1973; Rouhana, 2004). Los resultados señalan la existencia de memorias defensivas construidas socialmente y transmitidas generacionalmente (Halbacks, 2004; Páez et al., 1998; Rosa et

3 Palabra en euskera que significa 'lucha', utilizada para designar a las personas del MLNV. 
al., 2000), donde la simbología y la mitificación ocupan un lugar central (Brown, 1997; Zulaika, 1990, 2007). La memoria sobre el conflicto vinculada a la identidad y a posiciones políticas nacionalistas españolas, se asocia a la violencia de ETA (19682010), mientras que la memoria sobre el conflicto vinculada a la identidad y al nacionalismo vasco, aparece ligada a la historia de dominación política y cultural de España como estado liberal, con la importancia de las guerras carlistas del s. XIX', la guerra civil española (1936-1939) y la dictadura franquista (1939-1975).

Finalmente, los resultados también apuntan a la existencia de visones de la historia más complejas, basadas en interpretaciones que incluyen elementos de las dos memorias colectivas encontradas, sobre todo asociadas a ese continuo de posiciones intermedias que definen la polarización social relativa del País Vasco.

\section{Un clima emocional complejo}

Los conflictos intratables se caracterizan por una gran intensidad emocional y por del desarrollo de orientaciones emocionales colectivas (Bar-Tal, 2007). En el caso que nos ocupa, los resultados indican la existencia de un clima emocional complejo (Bar-Tal, Halperin, \& De Rivera, 2007; De Rivera, 1992; De Rivera, Kurrien, \& Olsen, 2007). Se infiere que el odio hacia España por parte del MLNV y hacia el nacionalismo vasco desde el Estado español, y del miedo hacia la violencia de ETA y del Estado, son emociones centrales en el desarrollo del conflicto. Por otro lado, los resultados señalan la existencia de emociones de pena y tristeza por el sufrimiento generado, la fractura social y la prolongación del conflicto. De igual modo, también indican la existencia de emociones de cansancio, hartazgo y aburrimiento ante la omnipresencia del conflicto o las actitudes del Estado y del MLNV y emociones de frustración, desesperanza e impotencia ante los discursos de la clase política, la desu-

4 Las guerras carlistas del s. XIX en España supusieron la abolición de las leyes históricas de los territorios vascos, los Fueros, con base en las cuales gestionaba su soberanía (véase Castells, 2003). nión entre nacionalistas vascos y la imposibilidad de desarrollar el proyecto independentista, y por la imposibilidad de poder contribuir a la paz desde la ciudadanía.

\section{Atractor 1. Las diferentes manifestaciones de violencia ejercidas por ETA y grupos afines, y por el Estado español, con sus procesos de sufrimiento y victimización asociados}

Los resultados indican que las diferentes manifestaciones de violencia directa ejercidas por ETA y el estado español durante la dictadura de Franco (1936-1975) y el periodo democrático (1976-), y la vulneración de derechos humanos, la victimización y el sufrimiento asociados a estas, son uno atractor principal del conflicto. P34 (mujer, educación, NEP) afirma que:

Por un lado puede estar por supuesto ETA, y por otro lado puede estar todo lo que fue la dictadura, la violencia de los $\mathrm{GAL}^{[5]}$ ya en democracia, o los temas de las torturas. Luego, añadido a eso, por ejemplo la dispersión ${ }^{[6]}$ (de los presos de ETA y de colectivos afines), que también es una forma de violencia.

Un aspecto central de este atractor es el impacto en la polarización social, y en los significados sobre la victimización. Los resultados apuntan a diferentes atribuciones sobre las víctimas, desde la inclusión de las víctimas de todos los perpetradores, a la mera inclusión de las del propio grupo, y de mecanismos sociales de apropiación del sufrimiento y negación de la victimización ajena, vinculados a procesos de deshumanización y exclusión moral (Bar-Tal, 2007; Haslam, 2006; Opotow, 2001). Asimismo, los resultados indican que desde las élites de los diferentes grupos se ha manipulado la victimización y el sufrimiento propio, con tal de reforzar la identificación, la cohesión y la lealtad hacia con el

5 Grupos Antiterroristas de Liberación. Agrupaciones parapoliciales que practicaron el denominado terrorismo de Estado contra ETA y su entorno, entre 1983 y 1987.

6 La dispersión de los presos de ETA significa que estos no cumplen sus condenas en las cárceles próximas a los domicilios de sus familiares, sino en otras cárceles españolas. 
propio grupo, o para lograr réditos políticos y electorales (Ramanathapillai, 2006). En este sentido, P35 (varón, Paz y DD. HH., NVM) señala:

Aquí el dolor está parcializado, yo soy preso y solo veo mi dolor. Si hablas de las víctimas tienes que hablar de los presos, y si hablas de los presos tienes que hablar de las víctimas. Se ve en la manipulación política de las asociaciones de presos, y se ve también en la manipulación política de las víctimas (de ETA).

\section{Atractor 2. La vulneración de las}

Necesidades Humanas Básicas

Siguiendo la perspectiva de las Necesidades Humanas Básicas (NHB) (Burton 1990a; Galtung, 2003; Kelman, 1997), los resultados apuntan a la existencia de vulneraciones de las NHB de seguridad, identidad colectiva, reconocimiento, dignidad, sentido de justicia, participación política, control y autonomía. La vulneración de la seguridad ha aparecido vinculada a la violencia de ETA y del Estado, en especial hacia las personas y grupos que han querido desarrollar actividades políticas, ya sea desde el ámbito político como desde otros ámbitos como el periodístico o el académico.

Por lo que se refiere a la necesidad de identidad colectiva, los resultados indican vulneraciones contra el desarrollo de la identidad vasca vinculadas al desarrollo de la cultura vasca, en especial del euske$\mathrm{ra}^{7}$, y vulneraciones contra la expresión social de la identidad española por temor a represalias sociales. P22 (mujer, Paz y DD. HH., NN), señala que:

En la raíz del problema está el tema identitario, de una identidad que no se respeta, que no se le da la salida que se le debería dar, y esto hace que se enquiste.

En relación con la necesidad de reconocimiento y dignidad, las narrativas señalan una falta de reconocimiento hacia las víctimas de ETA y del Estado para la recuperación de su dignidad como víctimas, aunque el desarrollo de políticas favora-

7 Lengua vasca. bles a las víctimas de ETA ha podido suponer un agravante para las víctimas de agentes del Estado o de la extrema derecha. Esta falta de reconocimiento aparece vinculada a la vulneración de la dignidad y del sentido de justicia. Por ello, P59 (varón, Paz y DD. HH., NN) apunta a que:

Las víctimas de ETA en una época determinada fueron abandonadas, de ETA y de todo el mundo habría que añadir. Esa teoría dice ahora tienen que pasar a un primer plano durante mucho tiempo y resarcirse. Lo que pasa que eso ha supuesto que se haya hecho nuevamente unas injusticias con otras víctimas que lo son también.

Por otro lado, los resultados también apuntan a una falta de reconocimiento de la identidad vasca como identidad de un pueblo diferenciado del pueblo español, y a la negación el derecho de autodeterminación como una vulneración de la necesidad de autonomía y control del propio destino.

\section{Atractor 3. La interdependencia negativa entre las identidades colectivas vasca y española}

Entendemos por interdependencia negativa de las identidades colectivas la percepción según la cual la supervivencia de la propia identidad colectiva solamente es posible a costa de la desaparición de la otra (Kelman, 1997, 1999; Rothman, 1997). P34 (mujer, educación, NEP) afirma que:

Hay unas personas viven de forma armoniosa vivir en España, sentirse español con la cultura española, y al mismo tiempo ser vasco con todo lo que puede ser la cultura vasca, y otras personas que lo viven como cosas antagonistas, como una incompatibilidad de identidades.

Los resultados muestran cómo la interdependencia negativa de las identidades colectivas se asocia a visiones monolíticas y narcisistas de las identidades, en los sectores ideológicos vasco y español más radicales, y al deseo de homogeneización, por lo que P21 (mujer, sanidad, NEP) indica que: 
Los nacionalistas radicales pretenden que todos seamos iguales, que todos seamos lo mismo, que todos pensemos lo mismo.

De igual modo, también se asocia a la obligación social de identificarse con una de las identidades nacionales.

Por otro lado, los resultados señalan la importancia de la manipulación de las identidades colectivas vasca y española por parte de las élites políticas; por ejemplo, P44 (varón, educación, NEP) dice que:

El problema nefasto que tenemos es que las relaciones de liderazgo político están excesivamente escoradas o asentadas en la base de que para conseguir poder hay que exacerbar las identidades colectivas del personal. Es una forma muy fácil de conseguir poder.

Los resultados también indican la vinculación entre identidades colectivas e ideologías políticas, con el énfasis en la necesidad de un crear o mantener el Estado nacional para el mantenimiento de la propia identidad, tanto por parte de los nacionalistas vascos como por parte de los nacionalistas españoles, todo ello con la finalidad de alcanzar o mantener el poder.

\section{Atractor 4. Cuestiones ideológicas y políticas}

En relación con las creencias e ideologías políticas (Bar-Tal, 2000; Martín-Baró, 2003; Van Dijk, 1999), los resultados apuntan a tres planteamientos políticos e ideológicos: el constitucionalista/centralista, el autonomista y el independentista, asentados en un continuo de posiciones cuyos polos son la defensa de la unidad de España ${ }^{8}$, y la defensa del derecho de autodeterminación para las 7 provincias vascas ${ }^{9}$. Así, los resultados señalan la existencia de

8 El Artículo 2 de la Constitución Española de 1978 dice: "La Constitución se fundamenta en la indisoluble unidad de la Nación española, patria común e indivisible de todos los españoles, y reconoce y garantiza el derecho a la autonomía de las nacionalidades y regiones que la integran y la solidaridad entre todas ellas".

9 Gipuzkoa, Bizkaia, Araba y Nafarroa en España. Lapurdi, Zuberoa y Nafarroa Behera en Francia. una contradicción nacional y de lucha por el poder con base en esa contradicción nacional. P35 (varón, Paz y DD. HH., NVM) afirma que:

Hay tres planteamientos, uno es el planteamiento constitucionalista, otro es el que dice que la constitución no la aceptamos pero aceptamos el estatuto de autonomía, y los que dicen aquí ni autonomía ni estatuto. Hay dos dogmas de fe en los que no se cede, la constitución española y el derecho de autodeterminación y la territorialidad.

En relación con los partidos políticos, los discursos señalan que han jugado un papel central en la perpetuación del conflicto, contribuyendo a generar división en la sociedad vasca a partir de actitudes partidistas vinculadas al logro del poder. Asimismo, apuntan a que los diferentes partidos políticos, tanto nacionalistas vascos ${ }^{10}$ como españoles ${ }^{11}$, han usado el conflicto con la pretensión de obtener réditos políticos y electorales, e incluso se advierte un interés en perpetuar el conflicto. Así, P58 (varón, periodismo, NVM) afirma que:

Yo he llegado a hablar con políticos y hay ciertos políticos que tienen interés en que las cosas no se resuelvan nunca. De todo, nacionalistas vascos y españoles, no me refiero solo a los nacionalistas españoles, sino incluso también te diría nacionalistas vascos.

Por último, se indica la importancia de las ideologías de izquierda y derecha, identificándose como polos del conflicto el independentismo vasco de extrema izquierda y el centralismo español de ideología conservadora.

\section{Atractor 5. Violencia cultural y legitimación de la violencia}

La violencia cultural y la legitimación de la violencia es un aspecto central y necesario para el ejerci-

10 PNV (Partido Nacionalistas Vasco)

11 PP, PSOE y UPyD (Partido Popular, Partido Socialista Obrero Español y Unión, Progreso y Democracia) 
cio de la violencia política (Barreto \& Borja, 2007; Barreto, Borja, Serrano, \& López-López, 2009; Berger, Ridgeway, Fisek, \& Norman, 1998; BorjaOrozco, Barreto, Sabucedo, \& López-López, 2008; De la Corte, Sabucedo, \& Blanco, 2004; Galtung, 1990; Kelman 2001). Un aspecto al que apuntan los resultados es la importancia de la construcción sociocultural, la transmisión generacional y los procesos de socialización en el desarrollo de las formas de legitimar la violencia de ETA y del Estado.

En relación con la violencia del Estado, los resultados muestran como la legitimación de la violencia aparece asociada a la necesidad de utilizar todos los recursos del Estado para acabar con ETA. P26 (varón, víctimas de ETA, NEP) afirma que: "Como reacción contraria pensemos en el GAL. Hay que reconocer que muchos contrarios a ETA eso lo veían bastante bien".

Por otro lado, señalan diferentes lógicas vinculadas a la legitimación de la violencia de ETA. En primer lugar, a la existencia de un conflicto político y armando provocado por el Estado español anterior a la existencia de ETA, y la existencia de una historia de dominación por parte del Estado español hacia las provincias vascas. En este sentido, P29 (mujer, Paz y DD. HH., NEP) dice.

Hay que resolver este conflicto porque por eso se mata. A mí ese discurso me parece perverso, porque es justificar que uno que se acerque a Juan ${ }^{12} \mathrm{y}$ le ponga una bomba al lado y lo mate.

Otra forma de legitimación ha sido la violencia utilizada por el Estado durante el periodo democrático (GAL, torturas, cárcel). En tercer lugar, se hace referencia a la lucha por la supervivencia de la identidad vasca en los territorios considerados como propios, con la centralidad de la supervivencia del euskera. P23 (varón, Paz y DD. HH., NEP) apunta que:

La sociedad vasca está penetrada por el conflicto de identidades, pero es un conflicto que no justifica de ningún modo una acción de violencia terrorista.

12 Se ha modificado el nombre original para el mantenimiento del anonimato.
Y por último, a las ideologías vinculadas al mito revolucionario y a la liberación nacional según las cuales las transformaciones políticas solo son posibles a través del uso de la violencia.

En otro orden de cosas, los resultados también indican cómo la perpetuación de la espiral de violencia ha estado asociada a procesos de exclusión moral (Opotow, 2001) y deshumanización (Haslam, 2006) de los miembros del exogrupo, en especial de las víctimas del exogrupo, y de procesos de desenganche moral (Bandura, 1999; Bandura et al., 1996) en relación con la violencia cometida por el propio grupo. P35 (varón, Paz y DD. HH., NVM) indica que:

Se dice que estos no tienen dolor, y con el dolor que tienen que se jodan. Unos dicen este era un fascista y lo han matado, pues bien, y los otros dicen este era un asesino terrorista y también.

\section{Discusión}

El modelo del conflicto profundo basado en el desarrollo del pensamiento complejo, nos ofrece una nueva óptica desde la que abordar el trabajo en resolución de conflictos y construcción de paz (Coleman, 2006; Coleman et al., 2007; Vallacher et al., 2010). Bajo este punto de vista, hemos intentado identificar los atractores (psicológicos, sociales y estructurales) del conflicto vasco, siguiendo la propuesta de Vallacher et al., es decir, analizando en qué medida los discursos de las personas se articulan alrededor de cuestiones centrales que se relacionan con otros elementos.

Los resultados nos sugieren que el conflicto vasco es un sistema complejo con atractores pertenecientes a diferentes dimensiones. En primer lugar, las diferentes formas de violencia directa ejercidas por ETA y el Estado español, y la espiral de violencia generada, se han erigido como el patrón de relaciones entre el MLNV y el Estado, y han mostrado una gran influencia en otras dimensiones del conflicto. A partir de diferentes informes sobre las consecuencias de la violencia política en el País Vasco desde el nacimiento de ETA (Asociación Pro Derechos Humanos. Argituz, 2011; Martín Beristain \& Páez, 
2000), se señala que ETA y grupos derivados ${ }^{13}$ han causado alrededor de 850 muertes y 1800 heridos, y más de 2000 personas han tenido que vivir con escolta. También se estima que alrededor de 100 personas han sido muertas y 650 heridas por las fuerzas de seguridad del Estado español, además de 80 muertes y 320 personas heridas por parte de grupos parapoliciales (GAL) y por el terrorismo tardo franquista ${ }^{14}$. También se señala que han sido detenidas, encarceladas, y en muchos casos en condiciones de aislamiento y dispersión, entre 10000 y 15000 , y que entre 5000 y 6000 pueden haber sido víctimas de malos tratos y tortura.

La perpetración de violencia política está siempre acompañada de lógicas justificadoras (De la Corte et al., 2004; Galtung, 1990); en este estudio vemos la presencia de diferentes formas de justificación de la violencia asociadas a memorias colectivas sobre el conflicto, a creencias sobre el propio grupo y el enemigo, y a procesos morales de exclusión, deshumanización y desenganche moral. Por ello, interpretamos que las formas de legitimación de la violencia son otro de los atractores del conflicto (Sabucedo et al., 2002; Sabucedo, Blanco, \& De la Corte, 2003; Van Den Broek, 2004; Varela-Rey, Rodríguez-Carballeira, Martín-Peña, 2013). Diversas investigaciones apuntan que la violencia de ETA se ha justificado sobre la base de una memoria donde las guerras del s. XIX y el s. XX adquieren un sentido glorioso y mitificado (Muro, 2009), a lógicas vinculadas a la identidad positiva del propio grupo y negativa del exogrupo, a una violencia ofensiva por parte del Estado y defensiva por parte de ETA y a una definición de la situación presente como injusta y de un futuro justo (Valera-Rey et al., 2013), a la existencia de un conflicto relevante para el grupo, la atribución de responsabilidades a otros, la deslegitimación de las víctimas y la atribución de ser víctimas del Estado (Sabucedo et al., 2002) y a procesos de exclusión moral (Martín-Peña \& Opotow, 2011).

13 ETA V, ETA VI, ETA militar, ETA político-militar, Comandos Autónomos Anticapitalistas.

14 Grupos parapoliciales de extrema derecha, tales como el Batallón Vasco Español o la Alianza Apostólica Anticomunista (Triple A), que atentaron contra miembros de ETA y su entorno, y contra otros grupos de izquierda en el estado español, entre 1976 y 1981.
Por otro lado, nos encontramos ante una vulneración de las distintas NHB interrelacionadas entre sí y con otros elementos del sistema/conflicto, por lo que consideramos dichas vulneraciones como un atractor del conflicto vasco. Entre ellas, la vulneración de la identidad colectiva se ha mostrado asociada a otro elemento considerado como un atractor del conflicto, como es la interdependencia negativa de las identidades colectivas (Kelman, 1999). El atractor identitario sugiere la existencia de una gran influencia de los procesos psicosociales vinculados al desarrollo de las identidades colectivas, en especial a la baja complejidad cognitiva, a las imágenes del Otro, a las memorias colectivas y las orientaciones emocionales colectivas (Bar-Tal, 2007). Los resultados señalan la centralidad del problema identitario en el conflicto vasco, un problema identitario de naturaleza política, donde las identidades etnonacionales están estrechamente vinculadas a las ideologías nacionalistas. Así, podemos advertir que la contradicción nacional inherente al conflicto vasco es otro atractor del conflicto, ya que los resultados nos indican la existencia de un desencuentro político histórico que ha condicionado la vida social y política del País Vasco a lo largo de la historia.

\section{Limitaciones del estudio}

La aproximación al conflicto vasco mediante el IAP (Smith, 2003, 2008; Smith et al., 1995) no nos permite generalizar los resultados obtenidos. La conclusiones parten de una interpretación doblemente hermenéutica de los resultados, al tratar de dar sentido al conjunto de significados sobre el fenómeno del conflicto vasco. Por ello, esta investigación no tiene la pretensión de ser exhaustiva, concluyente, ni generalizable, sino una aproximación al conflicto vasco con el modelo psicosocial dinámico sistémico del conflicto.

\section{Referencias}

Alvarez Enparantza, J. L. (1997). Euskal Herria en el horizonte. Tafalla: Txalaparta. 
Apalategi, J. (1992). Sociología de una nación en movimiento. Vitoria: Instituto de Estudios sobre Nacionalismos Comparados.

Apalategi, J. (2006). Los vascos, de la nación al Estado. Pamplona: Herritar Berri.

Aravena, A. (2003). El rol de la memoria colectiva y de la memoria individual en la conversión identitaria mapuche. Estudios Atacameños, 26, 89-96.

Asociación Pro Derechos Humanos. Argituz. (2011). Mapa (incompleto) de conculcaciones del derecho a la vida y a la integridad física y psíquica en relación a la violencia de motivación política. Extraído de http://www.argituz.org/documentos/inf/mapacastfinal.pdf

Azar, E. (1983). The theory of protracted social conflict and the challenge of transforming conflict situations. Monograph Series in World Affairs, 20(2), 81-99.

Azar, E. (1990). The management of protracted social conflict, theory and cases. Aldershot: Darmouth.

Bandura, A. (1999). Moral disengagement in the perpetration of inhumanities. Personality and Social Psychology Review, 3(3), 193-209.

Bandura, A., Barbaranelli, C., Caprara, G. V., \& Pastoreli, C. (1996). Mechanisms of moral disengagement in the exercise of moral agency. Journal of Personality and Social Psychology, 7(2), 364-374.

Bardin, L. (1996). Análisis de contenido. Madrid: Akal.

Bar-Tal, D. (2000). From intractable conflict through conflict resolution to reconciliation: Psychological analysis. Political Psychology, 21(2), 351-365.

Bar-Tal, D. (2007). Sociopsychological foundations of intractable conflict. American Behavioral Scientist, 50(11), 1430-1453.

Bar-Tal, D., Halperin, E., \& De Rivera, J. (2007). Collective emotions in conflict situations: Societal implications. Journal of Social Issues, 63(2), 441-469.

Bar-Tal, D., \& Rouhana, N. (1998). Psychological dynamics of intractable ethnonational conflict. American Psychologist, 53(7), 761-770.

Barreto, I., \& Borja, H. (2007). Violencia política: algunas consideraciones desde la psicología social. Diversitas. Perspectivas en Psicología, 3(1), 109-119.

Barreto, I., Borja, H., Serrano, Y., \& López-López, W. (2009). La legitimación como proceso en la violencia política, medios de comunicación y construc- ción de culturas de paz. Universitas Psychologica, 8(3), 737-748.

Berger, J., Ridgeway, C., Fisek, M. H., \& Norman, R. Z. (1998). The legitimation and delegitimation of power and prestige orders. American Sociological Review, 63(3), 379-405.

Bilbao, K. (1996). Crónica de una izquierda singular (De ETA-Berri a EMK/MC y a Zutik-Batzarre). Extraido de www.kepabilbao.com/descargas/

Cronicadeunaizquierdasingular.pdf

Blank, T., \& Schmidt, P. (2003). National identity in a united Germany: Nationalism or patriotism? An empirical test with representative data. Political Psychology, 24(2), 259-288.

Borja-Orozco, H., Barreto, I., Sabucedo, J. M., \& LópezLópez, W. (2008). Construcción del discurso deslegitimador del adversario: Gobierno y paramilitarismo en Colombia. Universitas Psychologica, 7(2), 571-583.

Bronfenbrenner, U. (1961). The mirror image in SovietAmerican relations: A social psychologist's report. Journal of Social Issues, 17(3), 45-56.

Brown, M. E. (1997). Causes and implications of ethnic conflict. En M. Gibernau \& J. Rex (Eds.), The ethnicity reader: Nationalism, multiculturalism and migration (pp. 80-100). Cambridge: Polity Press.

Bruner, J. (1991). Actos de significado. Madrid: Alianza.

Burton, J. W. (1987). Resolving deep-rooted conflict: A handbook. Lanham, MD: University Press of America.

Burton, J. W. (1990a). Conflict: Human needs theory. New York: St. Martin's Press.

Burton, J. W. (1990b). Conflict: Resolution and prevention. London: Macmillan Press.

Coleman, P. T. (2000). Intractable conflict. En M. Deutsch, P. T. Coleman \& E. C. Marcus (Eds.), The handbook of conflict resolution. Theory and practice (428-450). San Francisco: Jossey-Bass.

Coleman, P. T. (2003). Characteristics of protracted, intractable conflict: Toward the development of a metaframework-I. Peace and Conflict: Journal of Peace Psychology, 9(1), 1-37.

Coleman, P. T. (2004a). Paradigmatic framing of protracted, intractable conflict: Toward the development of a metaframework-II. Peace and Conflict: Journal of Peace Psychology, 10(3), 197-235. 
Coleman, P. T. (2004b). Polarized collective identities: A review and synthesis of the literature. New York: Columbia University.

Coleman, P. T. (2006). Conflict, complexity, and change: A meta-framework for addressing protracted, intractable conflicts - III. Peace and Conflict: Journal of Peace Psychology, 12(4), 325-348.

Coleman, P. T. (2012). Conclusion: The essence of peace? Toward a comprehensive and parsimonious model of sustainable peace. En P. T. Coleman \& M. Deutsch (Eds.), Psychological components of sustainable peace (pp. 353-369). New York: Springer.

Coleman, P. T., \& Lowe, J. K. (2007). Conflict, identity, and resilience: Negotiating collective identities within the Israeli and Palestinian diasporas. Conflict Resolution Quarterly, 24(3), 377-411.

Coleman, P. T., Vallacher, R., Nowak, A., \& Bui-Wrzosinska, L. (2007). Intractable conflict as an attractor: Presenting a dynamical model of conflict, escalation, and intractability. American Behavioral Scientist, 50(11), 1454-1475.

Community Boards \& Alzate, R. (2000). Transformación del conflicto: curriculum para bachillerato y secundaria. Bilbao. Editorial Mensajero.

Conway, L. G., Suedfeld, P., \& Tetlock, P. E. (2001). Integrative complexity and political decisions that lead to war or peace. En D. J. Christie, R. V. Wagner \& D. D. Winter (Eds.), Peace, conflict and violence: Peace psychology for the 21st century (pp. 102-109). Upper Saddle River, NJ: Prentice Hall.

Christie, D. J., Wagner, R. V., \& Winter, D. D. (2001). Peace, conflict and violence: Peace psychology for the 21 st century. Upper Saddle River, NJ: Prentice Hall.

De la Corte, L., Sabucedo, J. M., \& Blanco, A. (2004). Una función ética de la psicología social: los estudios sobre el fondo ideológico de la violencia política. Revista Interamericana de Psicología, 38(2), 171-180.

De Rivera, J. H. (1992). Emotional climate: Social structure and emotional dynamics. En K. T. Strongman (Ed.), International review of studies on emotion (pp. 197-218). New York: John Wiley.

De Rivera, J., Kurrien, R., \& Olsen, N. (2007). The emotional climate of nations and their culture of peace. Journal of Social Issues, 63(2), 255-271.
Deutsch, M. (1973). The resolution of conflict: Constructive and destructive processes. New Haven, CT: Yale University Press.

Dimitrov, V. (2000). Introduction to social fuzziology. En V. Dimitrov \& V. Korotkich (Eds.), Fuzzy logic: A framework for the new millennium. Heilderberg/ New York: Physica Verlag. Recuperado de: http:// www.zulenet.com/VladimirDimitrov/pages/IntroSocialFuzz.html

Dimitrov, V. (2003). Paradigm of complexity: The law of emergence. Recuperado de: http://www.zulenet. com/VladimirDimi trov/pages/paradigm.html

Doob, L. W. (1970). Resolving conflict in Africa: The Fermeda workshop. New Haven: Yale University Press.

Druckman, D. (2001). Nationalism and war: A socialpsychological perspective. En D. J. Christie, R. V. Wagner \& D. D. Winter (Eds.), Peace, conflict and violence: peace psychology for the 21st century (pp. 49-65). Upper Saddle River, NJ: Prentice Hall.

Euskadi Ta Askatasuna. (8 de enero, 2011). Declaración de ETA. Recuperado de: http://estaticos.elmundo. es/documentos/2011/01/10/comunicado_eta.pdf

Federico, C. M., Golec, A., \& Dial, J.L. (2005). The relationship between the need for closure and suport for military action against Iraq: Moderating effects of national attachment. Personality and Social Psychology Bulletin, 31(5), 621-632.

Feshback, S. (1994). Nationalism, patriotism and aggression: A clarification of functional differences. En L. R. Huesman (Ed.), Aggressive behavior: Current perspectives (pp. 275-291). New York: Plenum Press.

Fisher, R. J. (1990). The social psychology of intergroup and international conflict resolution. New York: Springer-Verlag.

Fisher, R. J. (1997). Interactive conflict resolution. New York: Syracuse University Press.

Galtung, J. (1969). Violence, peace and peace research. Journal of Peace Research, 6(3), 167-191.

Galtung, J. (1990). Cultural Violence. Journal of Peace Research, 27(3), 291-305.

Galtung, J. (2003). Paz por medios pacíficos. Paz y conflicto, desarrollo y civilización. Bilbao: Ed. Bakeaz/ Gernika Gogoratuz.

Goerner, S. (1995). Chaos, evolution and deep ecology. En R. Robertson \& A. Combs, Chaos theory in 
psychology and the live sciences (pp. 17-38). Hillsdale, NJ: Erlbaum.

Gurr, T. R. (2000). Peoples versus states: Minorities at risk in the new century. Washington, DC: United States Institute of Peace.

Haslam, N. (2006). Dehumanization: An integrative review. Personality and Social Psychology Review, 10(3), 252-264.

Halbwachs, M. (2004). La memoria colectiva. Zaragoza: Prensas Universitarias de Zaragoza.

Horowitz, D. L. (1985). Ethnic groups in conflict. Berkeley: University of California Press.

Hostil, O. R. (1969). Content analysis for the social sciences and humanities. Cambridge: Addison- Wesley.

Isajiw, W. W. (2000). Approaches to ethnic conflict resolution: Paradigms and principles. International Journal of Intercultural Relations, 24(1), 105-124.

Johnson, S. (2003). Sistemas emergentes. O iqué tienen en común hormigas, neuronas, ciudades y software? Madrid: Turner Publicaciones.

Kauffman, S. A. (1993). The origins of order: Self-organisation and selection in evolution. New York: Oxford University Press.

Kauffman, S. A. (1995). At home in the universe. The search for laws of complexity. London: Penguin Group.

Kelman, H. C. (1986). Interactive problem solving: A social-psychological approach to conflict resolution. En W. Klassen (Ed.), Dialogue toward inter-faith undestranding (pp. 293-314). Jerusalem: Ecumenical Institute for Theological Research. Kelman, H. C. (1992). Coalitions across conflict lines: The interplay of conflicts within and between the Israeli and Palestinian communities. En J. Simpson \& S. Worchel (Eds.), Conflict between people and peoples (pp. 165-190). Chicago: Nelson-Hall.

Kelman, H. C. (1997). Social-psychological dimensions of international conflict. En I. W.Zartman \&J. L. Rasmussen (Eds.), Peacemaking in international conflict. Methods Ë techniques (pp. 190-237). Washington: United States Institute or Peace Press.

Kelman, H. C. (1999). The interdependence of Israeli and Palestinian national identities: The role of the other in existential conflicts. Journal of Social Issues, 55(3), 581-600.
Kelman, H. C. (2001). The role of national identity in conflict resolution: Experiences from IsraeliPalestinian problem-solving workshops. En R. D. Ashmore, L. Jussim \& D. Wilder (Eds.), Social identity, intergroup conflict and conflict reduction (pp. 187-212). New York: Oxford University Press.

Korostelina, K. (2004). The impact of national identity on conflict behavior: Comparative analysis of two ethnic minorities in Crimea. International Journal of Comparative Sociology, 45(3-4), 213-230.

Korostelina, K. (2007). The system of social identities in Tajikistan: Early warning and conflict prevention. Communist and Post-Communist Studies, 40(2), 223-238.

Lederach, J. P. (1995). Preparing for peace. Conflict transformation across cultures. Syracuse: Syracuse University Press.

Lederach, J. P. (1998). Construyendo la paz. Reconciliación sostenible en sociedades divididas. Bilbao: Bakeaz y Gernika Gogoratuz.

Martín-Baró, I. (1983). Polarización social en El Salvador. San Salvador: Estudios Centroamericanos.

Martín-Baró, I. (2003). Poder, ideología y violencia. Madrid: Editorial Trotta.

Martín Beristain, C., \& Páez, D. (2000). Violencia, apoyo a las víctimas y reconstrucción del tejido social. Experiencias internacionales y el desafío vasco. Madrid: Editorial Fundamentos.

Martín Peña, J., \& Opotow, S. (2011). The legitimation of political violence: A case study of ETA in the Basque Country. Peace and Conflict: Journal of Peace Psychology, 12(2), 132-150.

Morales La Paz, L. R. (2003). Autoorganización y orden espontáneo idos caras de la misma moneda? En R. Rubio, F. J. Vázquez \& F. F. Muñoz (Coords.), Procesos de autoorganización (pp. 377-399). Madrid: Unión Editorial.

Morin, E. (1995). Introducción al pensamiento complejo. Barcelona: Gedisa.

Munné, F. (2005). ¿Qué es la complejidad? Número monográfico: La complejidad en la psicología social y de las organizaciones. Encuentros de Psicología Social, 3(2), 6-17. Recuperado de: http://www.portalpsicologia.org/servlet/File?idDocumento $=3384$ 
Muro, D. (2009). The politics of war memory in radical Basque nationalism. Ethnic and Racial Studies, 32(4), 659-678.

Musallam, N., Coleman, P. T., \& Nowak, A. (2010). Understanding the spread of malignant conflict: A dynamical-systems perspective. Peace and Conflict: The Journal of Peace Psychology, 16(2), 127-151.

Novak, A., Bui-Wrzosinska, L., Vallacher, R., \& Coleman, P. T. (2012). Sustainable peace: A dynamical systems perspective. En P. T. Coleman \& M. Deutsch (Eds.), Psychological components of sustainable peace (pp. 265-281). New York: Springer.

Opotow, S. (1990). Deterring moral exclusion. Journal of Social Issues, 46, 173-182.

Opotow, S. (2001). Social injustice. En D. J. Christie, R. V. Wagner \& D. D. Winter (Eds.), Peace, conflict and violence: Peace psychology for the 21st century (pp. 102-109). Upper Saddle River, NJ: Prentice Hall.

Opotow, S. (2005). Hate, conflict, and moral exclusion. En R. J. Sternberg (Ed.), The psychology of hate (pp. 121-153). Washington, DC: American Psychological Association.

Overschall, A. (2000). The manipulation of ethnicity: From ethnic cooperation to violence and war in Yugoslavia. Ethnic and Racial Studies, 23(6), $982-$ 1001.

Páez, D., Valencia, J. F., Pennebaker, J. W., Rimé, B., \& Jodelet, D. (1998). Memorias colectivas de procesos culturales y políticos. Bilbao: Servicio Editorial de la Universidad del País Vasco.

Pérez Martínez, A., Nuño de la Rosa, L., \& González Recio, J. L. (9-12 de enero de 2006). La obra de Stuart Kauffman. El problema del orden complejo y sus implicaciones filosóficas. Trabajo presentado en el Congreso sobre Complejidad: Implicaciones filosóficas, epistemológicas y metodológicas de la teoría de la complejidad. Instituto de Filosofía de la Universidad de La Habana, Cuba. Extraído de http://eprints.ucm.es/5120/

Ramanathapillai, R. (2006). The politicizing of trauma: A case study of Sri Lanka. Peace and Conflict: Journal of Peace Psychology, 12(1), 1-18.

Rosa Rivero, A., Bellelli, G., \& Bakhurst, D. (2000). Memoria colectiva e identidad nacional. Madrid: Biblioteca Nueva.
Ross, M. H. (1995). La cultura del conflicto. Las diferencias interculturales en la práctica de la violencia. Barcelona: Paidós.

Ross, M. H. (1997). The relevance of culture for the study of political psychology and ethnic conflict. Political Psychology, 18(2), 290-326.

Rothman, J. (1997). Resolving identity-based conflict in nations, organizations and communities. San Francisco: Jossey Bass.

Rouhana, N. N. (2004). Group identity and power asymmetry in reconciliation processes: The IsraeliPalestinian case. Peace and Conflict: Journal of Peace Psychology, 10(1), 33-52.

Rubin, J. Z., Pruitt, D. G., \& Kim, S. H. (1994). Social conflict: Escalation, stalemate and settlement. NewYork: McGraw-Hill.

Sabucedo, J. M., Blanco, A., \& De la Corte, L. (2003). Beliefs which legitimize political violence against the innocent. Psicothema, 15(4), 550-555.

Sabucedo, J. M., Rodríguez, M., \& Fernández, C. (2002). Construcción del discurso legitimador del terrorismo. Psicothema, 14(Supl.), 72-77.

Smith, A. D. (1993). The ethnic sources of nationalism. En M. E. Brown (Ed.), Ethnic conflict and international security (pp. 27-41). Princeton, NJ: Princeton University Press.

Smith, J. A. (2008). Qualitative psychology: A practical guide to research methods. London: Sage.

Smith, J. A., Harré, R., \& Van Langenhove, L. (1995). Rethinking methods in psychology. Londres: Sage.

Toscano, R. (1998). The face of the other: Ethics and intergroup conflict. En E. Weiner (Ed.), The handbook of interethnic coexistence (pp. 63-81). New York: Continuum.

Vallacher, R., Coleman, P. T., Nowak, A., \& Bui-Wrzosinska, L. (2010). Rethinking intractable conflict: The perspective of dynamical systems. American Psychologist, 65(4), 262-278.

Van Den Broek, H. (2004). Borroka. The legitimation of street violence in the political discourse of radical Basque nationalists. Terrorism and Political Violence, 16(4), 714-736.

Van Dijk, T. (1999). Ideología. Una aproximación multidisciplinaria. Barcelona: Gedisa.

Varela-Rey, A., Rodríguez-Carballeira, A., \& MartínPeña, J. (2013). Psychosocial analysis of ETA's vio- 
lence legitimation discourse. Revista de Psicología Social, 28(1), 85-97.

Worchel, S., \& Coutant, A. (1997). The tanget web of loyality: Nationalism, patriotism and ethnocentrism. En D. Bar-Tal \& E. Staub (Eds.), Patriotism in the lives of individuals and nations (pp. 190-210). Chicago: Nelson-Hall Publications.

Zulaika, J. (1990). Violencia vasca. Metáfora y sacramento. Madrid: Nerea.

Zulaika, J. (2007). Polvo de ETA. Irun: Alberdania. 